\title{
A LIE-TROTTER PRODUCT FORMULA FOR ORNSTEIN-UHLENBECK SEMIGROUPS IN INFINITE DIMENSIONS
}

\author{
FRANZISKA KÜHNEMUND AND JAN VAN NEERVEN
}

\begin{abstract}
We prove a Lie-Trotter product formula for the Ornstein-Uhlenbeck semigroup associated with the stochastic linear Cauchy problem

$$
\begin{aligned}
d X(t) & =A X(t) d t+d W(t), \quad t \geqslant 0, \\
X(0) & =x_{0} .
\end{aligned}
$$

Here $A$ is the generator of a $C_{0}$-semigroup on a separable real Banach space $E$ and $\{W(t)\}_{t \geqslant 0}$ is an $E$-valued Brownian motion.
\end{abstract}

\section{INTRODUCTION}

In this paper we prove a Lie-Trotter product formula for the Ornstein-Uhlenbeck semigroup associated with the stochastic linear Cauchy problem

$$
\begin{aligned}
d X(t) & =A X(t) d t+d W(t), \quad t \geqslant 0, \\
X(0) & =x_{0},
\end{aligned}
$$

where $A$ is the generator of a $C_{0}-$ semigroup $\{S(t)\}_{t \geqslant 0}$ on a separable real Banach space $E$ and $\{W(t)\}_{t \geqslant 0}$ is an $E$-valued Brownian motion. A predictable $E$-valued process $\left\{X\left(t, x_{0}\right)\right\}_{t \geqslant 0}$ is called a weak solution of (1.1) if for all $x^{*} \in \mathscr{D}\left(A^{*}\right)$ the process $\left\{\left\langle X\left(t, x_{0}\right), A^{*} x^{*}\right\rangle\right\}_{t \geqslant 0}$ is locally integrable almost surely and for all $t \geqslant 0$ we have, almost surely,

$$
\left\langle X\left(t, x_{0}\right), x^{*}\right\rangle=\left\langle x_{0}, x^{*}\right\rangle+\int_{0}^{t}\left\langle X\left(s, x_{0}\right), A^{*} x^{*}\right\rangle d s+\left\langle W(t), x^{*}\right\rangle .
$$

It is well-known [4] that (1.1) has a unique weak solution $\left\{X\left(t, x_{0}\right)\right\}_{t \geqslant 0}$ for some (hence, for all) $x_{0} \in E$ if and only if for all $t \geqslant 0$ the operator $Q_{t} \in \mathscr{L}\left(E^{*}, E\right)$ defined by

$$
Q_{t} x^{*}:=\int_{0}^{t} S(s) Q S^{*}(s) x^{*} d s, \quad x^{*} \in E^{*},
$$

is the covariance operator of a centred Gaussian measure on $E$; here $Q \in \mathscr{L}\left(E^{*}, E\right)$ is the covariance operator of the random variable $W(1)$. We then may define a one-parameter semigroup $\{\mathscr{P}(t)\}_{t \geqslant 0}$ of linear contractions on $C_{b}(E)$, the space of all bounded continuous real-valued functions on $E$, by

$$
\mathscr{P}(t) f(x):=\mathbb{E}(f(X(t, x))), \quad t \geqslant 0, x \in E .
$$

This semigroup is usually referred to as the transition semigroup or the OrnsteinUhlenbeck semigroup associated with equation (1.1). The random variables $X(t, x)$

2000 Mathematics Subject Classification. Primary: 35R15, 60H15; Secondary: 46D06, 60J35.

Key words and phrases. Ornstein-Uhlenbeck semigroup, Lie-Trotter product formula. 
are Gaussian with mean $S(t) x$ and covariance $Q_{t}$. Denoting by $\mu_{t}$ the centred Gaussian measure with covariance $Q_{t}$, we have

$$
\mathscr{P}(t) f(x)=\int_{E} f(S(t) x+y) d \mu_{t}(x), \quad t \geqslant 0, x \in E .
$$

In general, $\{\mathscr{P}(t)\}_{t \geqslant 0}$ fails to be strongly continuous in the supremum norm of $C_{b}(E)$. In this paper we consider $C_{b}(E)$ in its topology $\tau_{c}$ of uniform convergence on compact sets. In this topology, the Ornstein-Uhlenbeck semigroup is known to be strongly continuous [9]. We shall prove that under suitable conditions the following Lie-Trotter product formula holds:

$$
\mathscr{P}(t) f=\tau_{c}-\lim _{m \rightarrow \infty}\left[\mathscr{T}\left(\frac{t}{m}\right) \mathscr{S}\left(\frac{t}{m}\right)\right]^{m} f
$$

for all $f \in C_{b}(E)$, the convergence being uniform on every bounded time interval $[0, T]$. In this formula, $\{\mathscr{S}(t)\}_{t \geqslant 0}$ and $\{\mathscr{T}(t)\}_{t \geqslant 0}$ are the semigroups on $C_{b}(E)$ corresponding to the drift term and the diffusion term in (1.1). Thus,

$$
\begin{aligned}
& \mathscr{S}(t) f(x)=f(S(t) x), \\
& \mathscr{T}(t) f(x)=\int_{E} f(x+y) d \nu_{t}(y), \quad t \geqslant 0, x \in E,
\end{aligned}
$$

where $\nu_{t}$ is distribution of the random variable $W(t)$.

\section{Preliminaries}

In this preliminary section we recall some well-known facts about Gaussian measures and reproducing kernel Hilbert spaces. For more details we refer to the books $[2,18]$.

2.1. Gaussian measures. Let $E$ be a separable real Banach space. A Gaussian measure on $E$ is a Borel probability measure $\mu$ on $E$ with the property that for all $x^{*} \in E^{*}$ the image measure $\left\langle\mu, x^{*}\right\rangle:=\mu \circ x^{*-1}$ is Gaussian on $\mathbb{R}$. The mean of $\mu$ is defined by

$$
m:=\int_{E} x d \mu(x)
$$

this integral can be shown to converge absolutely in $E$. We call $\mu$ centred if $m=0$. If $\mu$ is a Gaussian measure on $E$ with mean $m$, there exists a unique compact operator $Q \in \mathscr{L}\left(E^{*}, E\right)$, the covariance operator of $\mu$, with the property that

$$
\left\langle Q x^{*}, y^{*}\right\rangle=\int_{E}\left\langle x-m, x^{*}\right\rangle\left\langle x-m, y^{*}\right\rangle d \mu(x), \quad x^{*}, y^{*} \in E^{*} .
$$

In terms of $m$ and $Q$, the Fourier transform of $\mu$ is given by

$$
\int_{E} \exp \left(-i\left\langle x, x^{*}\right\rangle\right) d \mu(x)=\exp \left(-i\left\langle m, x^{*}\right\rangle-\frac{1}{2}\left\langle Q x^{*}, x^{*}\right\rangle\right), \quad x^{*} \in E^{*} .
$$

Hence as a Gaussian measure, $\mu$ is determined uniquely by $m$ and $Q$. Sometimes we shall use the notation $N(m, Q)$ to denote the Gaussian measure with mean $m$ and covariance $Q$.

If $\{W(t)\}$ is an $E$-valued Brownian motion, then the distribution of the random variable $W(1)$ is a centred Gaussian measure on $E$. Denoting its covariance operator by $Q$, for all $s, t \geqslant 0$ and $x^{*}, y^{*} \in E^{*}$ we have

$$
\mathbb{E}\left(\left\langle W(s), x^{*}\right\rangle\left\langle W(t), y^{*}\right\rangle\right)=(s \wedge t)\left\langle Q x^{*}, y^{*}\right\rangle, \quad x^{*}, y^{*} \in E^{*} .
$$


Conversely, if $Q$ is the covariance operator of a Gaussian measure on $E$, there exist $E$-valued Brownian motions whose covariance is given by (2.3) [2, Proposition $7.2 .3]$.

Let $\mathscr{M}(E)$ denote the set of all Borel probability measures on $E$. Every $\mu \in$ $\mathscr{M}(E)$ determines a positive linear functional on $C_{b}(E)$ in a canonical way. The induced weak*-topology on $\mathscr{M}(E)$ is usually referred to as the weak topology of $\mathscr{M}(E)$.

Every measure $\mu \in \mathscr{M}(E)$ is tight, i.e., for every $\varepsilon>0$ there exists a compact subset $K \subseteq E$ such that $\mu(K) \geqslant 1-\varepsilon$. A family $\mathscr{M} \subseteq \mathscr{M}(E)$ is said to be tight if for every $\varepsilon>0$ there exists a compact subset $K \subseteq E$ such that $\mu(K) \geqslant 1-\varepsilon$ for all $\mu \in \mathscr{M}$. By Prohorov's theorem [2, Theorem 3.8.4], the family $\mathscr{M}$ is tight if and only if it is relatively compact with respect to the weak topology.

The covariance operator $Q$ of a Gaussian measure on $E$ is always positive, i.e.,

$$
\left\langle Q x^{*}, x^{*}\right\rangle \geqslant 0 \text { for all } x^{*} \in E^{*}
$$

and symmetric, i.e.,

$$
\left\langle Q x^{*}, y^{*}\right\rangle=\left\langle Q y^{*}, x^{*}\right\rangle \text { for all } x^{*}, y^{*} \in E^{*} .
$$

The converse does not hold: not every positive symmetric operator $Q \in \mathscr{L}\left(E^{*}, E\right)$ is the covariance operator of some Gaussian measure. In this connection the following result, which is a special case of [2, Theorem 3.3.6], will be useful:

Proposition 2.1. Let $R \in \mathscr{L}\left(E^{*}, E\right)$ be the covariance operator of a Gaussian measure on $E$. Let $\mathscr{Q} \subseteq \mathscr{L}\left(E^{*}, E\right)$ be a family of positive symmetric operators. If there exists a constant $C \geqslant 0$ such that

$$
\left\langle Q x^{*}, x^{*}\right\rangle \leqslant C\left\langle R x^{*}, x^{*}\right\rangle
$$

for all $x^{*} \in E^{*}$ and $Q \in \mathscr{Q}$, then every $Q \in \mathscr{Q}$ is the covariance of a centred Gaussian measure $\mu_{Q}$ on E. Moreover, the family $\left\{\mu_{Q}: Q \in \mathscr{Q}\right\}$ is tight.

The following result is concerned with weak convergence of sequences of Gaussian measures [2, Theorem 3.8.9].

Proposition 2.2. Let $\left(m_{n}\right)$ be a sequence in $E$ and $\left(Q_{n}\right)$ a sequence of covariance operators in $\mathscr{L}\left(E^{*}, E\right)$. For each $n$, put $\nu_{n}:=N\left(0, Q_{n}\right)$ and $\mu_{n}:=N\left(m_{n}, Q_{n}\right)$. Let further an element $m \in E$ and a covariance operator $Q \in \mathscr{L}\left(E^{*}, E\right)$ be given, and put $\nu:=N(0, Q)$ and $\mu:=N(m, Q)$. Then the following assertions are equivalent.

(1) $\lim _{n \rightarrow \infty} \mu_{n}=\mu$ weakly.

(2) $\lim _{n \rightarrow \infty} m_{n}=m$ strongly and $\lim _{n \rightarrow \infty} \nu_{n}=\nu$ weakly.

In this situation, for all $x^{*}, y^{*} \in E^{*}$ we have

$$
\lim _{n \rightarrow \infty}\left\langle Q_{n} x^{*}, y^{*}\right\rangle=\left\langle Q x^{*}, y^{*}\right\rangle \text {. }
$$

Let us now assume that $E$ is a separable real Hilbert space with inner product $[\cdot, \cdot]_{E}$. Identifying $E^{*}$ with $E$ in the canonical way, positive symmetric operators from $E^{*}$ into $E$ can be identified with positive selfadjoint operators on $E$. Under this identification, Such an operator $Q$ is the covariance of a centred Gaussian measure $\mu$ on $E$ if and only if it is a trace class operator. Moreover, if $\left\{e_{k}\right\}_{k=1}^{\infty}$ is an orthonormal basis for $E$, then by (2.1) we have

$$
\operatorname{tr} Q=\sum_{k=1}^{\infty}\left[Q e_{k}, e_{k}\right]_{E}=\int_{E} \sum_{k=1}^{\infty}\left[x, e_{k}\right]_{E}^{2} d \mu(x)=\int_{E}\|x\|^{2} d \mu(x) .
$$


Proposition 2.3. Let $E$ be a separable real Banach space and let $\left(\mu_{n}\right)$ be a sequence of centred Gaussian measures on $E$ with covariances $\left(Q_{n}\right)$. Let $\mu$ be a centred Gaussian measure on $E$ with covariance $Q$.

(i) If $\lim _{n \rightarrow \infty} \mu_{n}=\mu$ weakly, then:

(1) $\lim _{n \rightarrow \infty}\left[Q_{n} x, y\right]_{E}=[Q x, y]_{E}$ for all $x, y \in E$,

(2) $\lim _{n \rightarrow \infty} \int_{E}\|x\|^{2} d \mu_{n}(x)=\int_{E}\|x\|^{2} d \mu(x)$.

(ii) If $E$ is a separable real Hilbert space, then conversely the conditions (1) and (2) imply that $\lim _{n \rightarrow \infty} \mu_{n}=\mu$ weakly.

In (i), (1) follows by considering Fourier transforms and (2) is a special case of $[2$, Lemma 3.8.7]. In a formulation where (1) is replaced by a slightly stronger hypothesis, the converse assertion (ii) is proved in [2, Example 3.8.15]. For the convenience of the reader we include a proof of (ii) in its present formulation in the Appendix.

2.2. Reproducing kernel Hilbert spaces. We return to the situation where $E$ is a separable real Banach space. Let $Q \in \mathscr{L}\left(E^{*}, E\right)$ be an arbitrary positive symmetric operator. The mapping

$$
\left(Q x^{*}, Q y^{*}\right) \mapsto\left\langle Q x^{*}, y^{*}\right\rangle, \quad x^{*}, y^{*} \in E^{*},
$$

defines an inner product on the range of $Q$. The completion of range $(Q)$ with respect to this inner product is a separable real Hilbert space $H_{Q}$, the reproducing kernel Hilbert space (RKHS) associated with $Q$. The inclusion mapping from range $(Q)$ into $E$ extends to a continuous inclusion mapping $i_{Q}: H_{Q} \hookrightarrow E$. We have the operator identity

$$
Q=i_{Q} \circ i_{Q}^{*}
$$

The following simple observation will be useful in the next section.

Lemma 2.4. Let $Q, R \in \mathscr{L}\left(E^{*}, E\right)$ be positive and symmetric operators and assume that $H_{Q} \subseteq H_{R}$ as subsets of $E$. Then the inclusion mapping $i_{Q, R}$ from $H_{Q}$ into $H_{R}$ is bounded, and for all $x^{*} \in E^{*}$ we have

$$
\left\langle Q x^{*}, x^{*}\right\rangle \leqslant\left\|i_{Q, R}\right\|^{2}\left\langle R x^{*}, x^{*}\right\rangle .
$$

Proof. First we claim that $i_{Q, R}$ is closed. Indeed, suppose that $h_{n} \rightarrow h$ in $H_{Q}$ and $i_{Q, R} h_{n} \rightarrow \tilde{h}$ in $H_{R}$, Then $i_{Q} h_{n} \rightarrow i_{Q} h$ in $E$ and also $i_{Q} h_{n}=i_{R} i_{Q, R} h_{n} \rightarrow i_{R} \tilde{h}$ in $E$. Hence $i_{Q} h=i_{R} \tilde{h}$ in $E$. But also, $i_{Q} h=i_{R} i_{Q, R} h$, and therefore $\tilde{h}=i_{Q, R} h$, by the injectivity of $i_{R}$. This proves the claim. Boundedness of $i_{Q, R}$ is now an immediate consequence of the closed graph theorem.

Next, for all $x^{*} \in E^{*}$ and all $h \in H_{Q}$ we have

$$
\begin{aligned}
\left|\left[h, i_{Q}^{*} x^{*}\right]_{H_{Q}}\right| & =\left|\left\langle i_{Q} h, x^{*}\right\rangle\right|=\left|\left\langle i_{R} i_{Q, R} h, x^{*}\right\rangle\right|=\left|\left[i_{Q, R} h, i_{R}^{*} x^{*}\right]_{H_{R}}\right| \\
& \leqslant\left\|i_{Q, R} h\right\|_{H_{R}}\left\|i_{R}^{*} x^{*}\right\|_{H_{R}} \leqslant\left\|i_{Q, R}\right\|\|h\|_{H_{Q}}\left\|i_{R}^{*} x^{*}\right\|_{H_{R}} .
\end{aligned}
$$

Taking the supremum over all $h \in H_{Q}$ with $\|h\|_{H_{Q}} \leqslant 1$ we obtain $\left\|i_{Q}^{*} x^{*}\right\|_{H_{Q}} \leqslant$ $\left\|i_{Q, R}\right\|\left\|i_{R}^{*} x^{*}\right\|_{H_{R}}$, and hence

$$
\left\langle Q x^{*}, x^{*}\right\rangle=\left\|i_{Q}^{*} x^{*}\right\|_{H_{Q}}^{2} \leqslant\left\|i_{Q, R}\right\|^{2}\left\|i_{R}^{*} x^{*}\right\|_{H_{R}}^{2}=\left\|i_{Q, R}\right\|^{2}\left\langle R x^{*}, x^{*}\right\rangle .
$$


If $E$ is a separable real Hilbert space and $Q$ is a positive selfadjoint operator on $E$, then the RKHS associated with $Q$ equals range $\left(Q^{\frac{1}{2}}\right)$ with inner product

$$
\left[Q^{\frac{1}{2}} x, Q^{\frac{1}{2}} y\right]_{H_{Q}}=[x, y]_{E}, \quad x, y \in E .
$$

\section{The Lie-Trotter product Formula}

For the rest of the paper we will make the following standing assumption.

\section{Assumption 3.1.}

(1) $\{S(t)\}_{t \geqslant 0}$ is a $C_{0}$-semigroup on a separable real Banach space $E$.

(2) $Q \in \mathscr{L}\left(E^{*}, E\right)$ is the covariance of a centred Gaussian measure $\nu$ on $E$.

(3) For all $t \geqslant 0$, the operator $Q_{t} \in \mathscr{L}\left(E^{*}, E\right)$ defined by

$$
Q_{t} x^{*}:=\int_{0}^{t} S(s) Q S^{*}(s) x^{*} d s, \quad x^{*} \in E^{*},
$$

is the covariance of a centred Gaussian measure $\mu_{t}$ on $E$.

In the following situations, (3) automatically follows from (1) and (2).

- If $\{S(t)\}_{t \geqslant 0}$ restricts to a $C_{0}$-semigroup on the RKHS $H_{Q}$ associated with $Q$. This is an easy consequence of Proposition 2.1; cf. [12].

- If $E$ has type 2 (in particular, if $E$ is a Hilbert space) [14]. For the special case of $M$-type 2 spaces a more general result was proved by Brzeźniak [3, Section 2].

Let us pause to make a number of simple observations. First, by the positivity of $Q$, for all $0 \leqslant s \leqslant t$ and $x^{*} \in E^{*}$ we have

$$
\begin{aligned}
0 \leqslant\left\langle Q_{s} x^{*}, x^{*}\right\rangle & =\int_{0}^{s}\left\langle Q S^{*}(\sigma) x^{*}, S^{*}(\sigma) x^{*}\right\rangle d \sigma \\
& \leqslant \int_{0}^{t}\left\langle Q S^{*}(\sigma) x^{*}, S^{*}(\sigma) x^{*}\right\rangle d \sigma=\left\langle Q_{t} x^{*}, x^{*}\right\rangle .
\end{aligned}
$$

Next, for all $s, t \geqslant 0$ and $x^{*} \in E^{*}$ we have

$$
Q_{t+s} x^{*}=Q_{t} x^{*}+S(t) Q_{s} S^{*}(t) x^{*}
$$

and therefore

$$
\mu_{t+s}=\mu_{t} * S(t) \mu_{s}
$$

where the $*$ denotes convolution and $S(t) \mu_{s}:=\mu_{s} \circ S(t)^{-1}$ denotes the image measure.

We define linear contractions $\mathscr{P}(t)$ on $C_{b}(E)$ by

$$
\mathscr{P}(t) f(x):=\int_{E} f(S(t) x+y) d \mu_{t}(y), \quad x \in E, \quad t \geqslant 0 .
$$

It is an easy consequence of (3.2) that the family $\{\mathscr{P}(t)\}_{t \geqslant 0}$ is a semigroup on $C_{b}(E)$. In general, this semigroup fails to be strongly continuous in the supremum norm, even on the closed invariant subspace $B U C(E)$ of bounded uniformly continous functions on $E$. In fact, $\{\mathscr{P}(t)\}_{t \geqslant 0}$ is strongly continuous on $B U C(E)$ if and only if $A=0$, i.e., if the drift term is trivial $[15,13]$. For this reason many authors have studied strong continuity of $\{\mathscr{P}(t)\}_{t \geqslant 0}$ in various locally convex topologies on $C_{b}(E)$, cf. [5], [6], [8], [11], [16]. They only consider the situation where $E$ is a 
Hilbert space, in which case Itô calculus may be applied. Using analytic methods, the Banach space case was studied in [10], [12], [13], [9].

We will need the following result from [13], which is an easy consequence of Proposition 2.1 and (3.1).

Proposition 3.2. We have $\lim _{t \downarrow 0} \mu_{t}=\mu_{0}=\delta_{0}$ weakly, where $\delta_{0}$ is the Dirac measure on $E$ concentrated at 0 .

For the proof of the Lie-Trotter product formula it will be necessary to study tightness of a family of measures that is obtained by 'discretizing' the covariance operators of the measures $\mu_{t}$.

Let $P=\left\{t_{0}, \ldots, t_{N}\right\}$ be a partition of the interval $[0, t]$; i.e., $0=t_{0}<\cdots<t_{N}=$ $t$. We define positive symmetric operators $Q_{t}^{P} \in \mathscr{L}\left(E^{*}, E\right)$ by

$$
Q_{t}^{P} x^{*}:=\sum_{j=1}^{N}\left(t_{j}-t_{j-1}\right) S\left(t_{j}\right) Q S^{*}\left(t_{j}\right) x^{*}, \quad x^{*} \in E^{*} .
$$

Note that the sum defining $Q_{t}^{P} x^{*}$ is the Riemann sum for the integral

$$
Q_{t} x^{*}=\int_{0}^{t} S(s) Q S^{*}(s) x^{*} d s
$$

corresponding with the right endpoints of the partition intervals.

For every partition $P$ of $[0, t]$, the operator $Q_{t}^{P}$ is the covariance of a centred Gaussian measure $\mu_{t}^{P}$ on $E$. To see this, first note that for all $\lambda_{j}>0$, the operator $R_{j}:=\lambda_{j} Q$ is the covariance of the scaled measure $\nu_{j}(B):=\nu\left(B / \sqrt{\lambda_{j}}\right), B \subseteq E$ Borel. Next, if $R_{j}$ is the covariance of a centred Gaussian measure $\nu_{j}$ on $E$ and if $S_{1}, \ldots, S_{N}$ are bounded operators on $E$, then $\sum_{j=1}^{N} S_{j} R_{j} S_{j}^{*}$ is the covariance of the centred Gaussian measure $S_{1} \nu_{1} * \cdots * S_{N} \nu_{N}$. We finally apply this with $\lambda_{j}=t_{j}-t_{j-1}$ and $S_{j}=S\left(t_{j}\right)$.

The mesh of a partition $P$ is the number mesh $(P):=\max _{j=1, \ldots, N}\left(t_{j}-t_{j-1}\right)$.

Lemma 3.3. Let $\left(t_{n}\right)$ be a sequence of strictly positive real numbers satisfying $\lim _{n \rightarrow \infty} t_{n}=t$. For each $n$ let $P_{n}$ be a partition of $\left[0, t_{n}\right]$, and assume that $\lim _{n \rightarrow \infty} \operatorname{mesh}\left(P_{n}\right)=0$. Then, for all $x^{*}, y^{*} \in E^{*}$, we have

$$
\lim _{n \rightarrow \infty}\left\langle Q_{t_{n}}^{P_{n}} x^{*}, y^{*}\right\rangle=\left\langle Q_{t} x^{*}, y^{*}\right\rangle
$$

Proof. Fix $x^{*}, y^{*} \in E$. Being a Gaussian covariance operator, $Q$ is compact and therefore the function

$$
\phi(s):=\left\langle S(s) Q S^{*}(s) x^{*}, y^{*}\right\rangle=\left\langle Q S^{*}(s) x^{*}, S^{*}(s) y^{*}\right\rangle, \quad s \in[0, \infty),
$$

is continuous for all $x^{*} \in E^{*}$. Indeed, this follows from the weak ${ }^{*}$-continuity of the adjoint semigroup $\left\{S^{*}(t)\right\}_{t \geqslant 0}$ which is uniform on compact subsets of $E$.

Fix $\varepsilon>0$ arbitrary and fix $T>0$ large enough such that $0 \leqslant t_{n} \leqslant T$ for all $n$. The uniform continuity of $\phi$ on $[0, T]$ enables us to find $\delta>0$ small enough such that $\left|\phi(s)-\phi\left(s^{\prime}\right)\right|<\varepsilon / T$ for all $s, s^{\prime} \in[0, T]$ with $\left|s-s^{\prime}\right|<\delta$. Choose $N$ so large that mesh $\left(P_{n}\right)<\delta$ for all $n \geqslant N$. Then, for all $n \geqslant N$ we have

$$
\left|\left\langle Q_{t_{n}}^{P_{n}} x^{*}, y^{*}\right\rangle-\int_{0}^{t_{n}} \phi(s) d s\right|<\varepsilon .
$$


Therefore,

$\left|\left\langle Q_{t_{n}}^{P_{n}} x^{*}, y^{*}\right\rangle-\left\langle Q_{t} x^{*}, y^{*}\right\rangle\right|=\left|\left\langle Q_{t_{n}}^{P_{n}} x^{*}, y^{*}\right\rangle-\int_{0}^{t} \phi(s) d s\right|<\varepsilon+\left|t-t_{n}\right| \cdot \sup _{s \in[0, T]}|\phi(s)|$.

From this we conclude that

$$
\limsup _{n \rightarrow \infty}\left|\left\langle Q_{t_{n}}^{P_{n}} x^{*}, y^{*}\right\rangle-\left\langle Q_{t} x^{*}, y^{*}\right\rangle\right|<\varepsilon .
$$

Since $\varepsilon>0$ was arbitrary, this proves the lemma.

We define semigroups $\{\mathscr{S}(t)\}_{t \geqslant 0}$ and $\{\mathscr{T}(t)\}_{t \geqslant 0}$ on $C_{b}(E)$ by

$$
\begin{aligned}
\mathscr{S}(t) f(x) & :=f(S(t) x), \\
\mathscr{T}(t) f(x) & :=\int_{E} f(x+y) d \nu_{t}(y), \quad t \geqslant 0, x \in E,
\end{aligned}
$$

where $\nu_{t}$ denotes the centred Gaussian measure on $E$ with covariance $t Q$. The first semigroup, $\{\mathscr{S}(t)\}_{t \geqslant 0}$, can be interpreted as the transition semigroup corresponding to the deterministic equation

$$
d X(t)=A X(t) d t
$$

The second semigroup, $\{\mathscr{T}(t)\}_{t \geqslant 0}$, can be interpreted as the transition semigroup corresponding to the equation

$$
d X(t)=d W(t)
$$

assuming that $\{W(t)\}_{t \geqslant 0}$ is an $E$-valued Brownian motion such that for all $t \geqslant 0$ the random variable $W(t)$ has distribution $\nu_{t}$. Comparing this with (1.1), we see that equations (3.5) and (3.6) correspond to the drift term and the diffusion term in (1.1), respectively.

Our main abstract result relates the transition semigroup $\{\mathscr{P}(t)\}_{t \geqslant 0}$ to the semigroups $\{\mathscr{S}(t)\}_{t \geqslant 0}$ and $\{\mathscr{T}(t)\}_{t \geqslant 0}$.

Theorem 3.4. Let $\left(t_{n}\right)$ be a sequence of strictly positive real numbers satisfying $\lim _{n \rightarrow \infty} t_{n}=t$. For each $n$ let $P_{n}$ be a partition of $\left[0, t_{n}\right]$, and assume that $\lim _{n \rightarrow \infty} \operatorname{mesh}\left(P_{n}\right)=0$. Write $P_{n}=\left\{t_{0, n}, \ldots, t_{N_{n}, n}\right\}$, and for $j=1, \ldots, N_{n}$ put $\Delta t_{j, n}:=t_{j, n}-t_{j-1, n}$ and

$$
\mathscr{V}\left(\Delta t_{j, n}\right):=\mathscr{T}\left(\Delta t_{j, n}\right) \circ \mathscr{S}\left(\Delta t_{j, n}\right) .
$$

If

$$
\lim _{n \rightarrow \infty} \mu_{t_{n}}^{P_{n}}=\mu_{t} \quad \text { weakly }
$$

then for all $f \in C_{b}(E)$ and all sequences $\left(x_{n}\right)$ in $E$ with $\lim _{n \rightarrow \infty} x_{n}=x$ we have

$$
\mathscr{P}(t) f(x)=\lim _{n \rightarrow \infty}\left[\mathscr{V}\left(\Delta t_{N_{n}, n}\right) \circ \cdots \circ \mathscr{V}\left(\Delta t_{1, n}\right)\right] f\left(x_{n}\right) .
$$

Remark 3.5. In Sections 4 and 5 below we will show that condition (3.7) is automatically satisfied in each of the following two situations:

- $\{S(t)\}_{t \geqslant 0}$ restricts to a $C_{0}$-semigroup on the RKHS $H_{Q}$.

- $E$ is a Hilbert space. 
Proof of Theorem 3.4. Fix $f \in C_{b}(E)$ and $\xi \in E$. For all $s \geqslant 0$ we have

$$
[\mathscr{T}(s) \circ \mathscr{S}(s)] f(\xi)=\int_{E} \mathscr{S}(s) f(\xi+y) d \nu_{s}(y)=\int_{E} f(S(s) \xi+S(s) y) d \nu_{s}(y) .
$$

Writing $\nu_{s}^{t}$ for the image measure $S(t) \nu_{s}$, for all $s_{1}, s_{2} \geqslant 0$ we obtain

$$
\begin{aligned}
{\left[\mathscr{T}\left(s_{2}\right) \circ\right.} & \left.\mathscr{S}\left(s_{2}\right)\right] \circ\left[\mathscr{T}\left(s_{1}\right) \circ \mathscr{S}\left(s_{1}\right)\right] f(\xi) \\
& =\int_{E}\left[\mathscr{T}\left(s_{1}\right) \circ \mathscr{S}\left(s_{1}\right)\right] f\left(S\left(s_{2}\right) \xi+S\left(s_{2}\right) y\right) d \nu_{s_{2}}(y) \\
& =\int_{E} \int_{E} f\left(S\left(s_{1}+s_{2}\right) \xi+S\left(s_{1}+s_{2}\right) y+S\left(s_{1}\right) z\right) d \nu_{s_{1}}(z) d \nu_{s_{2}}(y) \\
& =\int_{E} \int_{E} f\left(S\left(s_{1}+s_{2}\right) \xi+\eta+\zeta\right) d \nu_{s_{1}}^{s_{1}}(\zeta) d \nu_{s_{2}}^{s_{1}+s_{2}}(\eta) \\
& =\int_{E} f\left(S\left(s_{1}+s_{2}\right) \xi+\varrho\right) d\left(\nu_{s_{1}}^{s_{1}} * \nu_{s_{2}}^{s_{1}+s_{2}}\right)(\varrho)
\end{aligned}
$$

By induction, for all $s_{1}, \ldots, s_{N} \geqslant 0$ we obtain

$$
\begin{aligned}
{\left[\mathscr{T}\left(s_{N}\right) \circ\right.} & \left.\mathscr{S}\left(s_{N}\right)\right] \circ \cdots \circ\left[\mathscr{T}\left(s_{1}\right) \circ \mathscr{S}\left(s_{1}\right)\right] f(\xi) \\
& =\int_{E} f\left(S\left(s_{1}+\cdots+s_{N}\right) \xi+\varrho\right) d\left(\nu_{s_{1}}^{s_{1}} * \cdots * \nu_{s_{N}}^{s_{1}+\cdots+s_{N}}\right)(\varrho) .
\end{aligned}
$$

Let us now fix a partition $P=\left\{\tau_{0}, \ldots, \tau_{N}\right\}$ of an interval $[0, \tau]$, take $s_{j}=\Delta \tau_{j}:=$ $\tau_{j}-\tau_{j-1}$ in the identity above and note that $\Delta \tau_{1}+\cdots+\Delta \tau_{k}=\tau_{k}$ for $k=1, \ldots, N$. The covariance operator of $\nu_{\Delta \tau_{1}}^{\tau_{1}} * \cdots * \nu_{\Delta \tau_{N}}^{\tau_{N}}$ equals

$$
\sum_{j=1}^{N} S\left(\tau_{j}\right)\left(\Delta \tau_{j} Q\right) S^{*}\left(\tau_{j}\right)=Q_{\tau}^{P} .
$$

Thus, we obtain

$$
\begin{aligned}
{\left[\mathscr{V}\left(\Delta \tau_{N}\right) \circ \cdots \circ \mathscr{V}\left(\Delta \tau_{1}\right)\right] f(\xi) } & =\int_{E} f\left(S\left(\tau_{N}\right) \xi+\varrho\right) d\left(\nu_{\Delta \tau_{1}}^{\tau_{1}} * \cdots * \nu_{\Delta \tau_{N}}^{\tau_{N}}\right)(\varrho) \\
& =\int_{E} f(S(\tau) \xi+\varrho) d \mu_{\tau}^{P}(\varrho) .
\end{aligned}
$$

After these preparations we turn to the proof of (3.8). Let $N(m, R)$ denote the Gaussian measure on $E$ with mean $m$ and covariance $R$. If $\lim _{n \rightarrow \infty} x_{n}=x$ in $E$, then by (3.7) and Proposition 2.2, we have

$$
\lim _{n \rightarrow \infty} N\left(S\left(t_{n}\right) x_{n}, Q_{t_{n}}^{P_{n}}\right)=N\left(S(t) x, Q_{t}\right) \quad \text { weakly. }
$$

It follows that

$$
\begin{aligned}
\lim _{m \rightarrow \infty}\left[\mathscr{V}\left(\Delta t_{N_{n}, n}\right) \circ \cdots \circ \mathscr{V}\left(\Delta t_{1, n}\right)\right] f\left(x_{n}\right) & =\lim _{n \rightarrow \infty} \int_{E} f\left(S\left(t_{n}\right) x_{n}+\varrho\right) d \mu_{t_{n}}^{P_{n}}(\varrho) \\
& =\int_{E} f(S(t) x+\varrho) d \mu_{t}(\varrho)=\mathscr{P}(t) f(x) .
\end{aligned}
$$

This proves (3.8).

From this result we deduce the following Lie-Trotter product formula for the semigroup $\{\mathscr{P}(t)\}_{t \geqslant 0}$ : 
Theorem 3.6. For $t>0$, let $\mu_{t, n}:=\mu_{t}^{\pi_{n}}$, where $\pi_{n}$ is the equipartition of $[0, t]$ into $n$ subintervals of equal length. If for all $t>0$ we have

$$
\lim _{n \rightarrow \infty} \mu_{t, n}=\mu_{t} \quad \text { weakly, }
$$

then for all $f \in C_{b}(E)$ and all $t \geqslant 0$ and $x \in E$ we have

$$
\mathscr{P}(t) f(x)=\lim _{n \rightarrow \infty}\left[\mathscr{T}\left(\frac{t}{n}\right) \circ \mathscr{S}\left(\frac{t}{n}\right)\right]^{n} f(x),
$$

the convergence being uniform on finite time intervals $[0, T]$ and compact subsets $K \subseteq E$.

Proof. Suppose (3.9) holds but (3.10) fails. We will deduce a contradiction as follows.

By assumption there exist an $\varepsilon>0$, a compact set $K \subseteq E$, a real number $T>0$, and a subsequence $\left(n_{k}\right)$ such that

$$
\sup _{(t, x) \in[0, T] \times K}\left|\mathscr{P}(t) f(x)-\left[\mathscr{T}\left(\frac{t}{n_{k}}\right) \circ \mathscr{S}\left(\frac{t}{n_{k}}\right)\right]^{n_{k}} f(x)\right| \geqslant \varepsilon
$$

for all $k$. Thus, we can choose points $\left(t_{k}, x_{k}\right) \in[0, T] \times K$ such that

$$
\left|\mathscr{P}\left(t_{k}\right) f\left(x_{k}\right)-\left[\mathscr{T}\left(\frac{t_{k}}{n_{k}}\right) \circ \mathscr{S}\left(\frac{t_{k}}{n_{k}}\right)\right]^{n_{k}} f\left(x_{k}\right)\right| \geqslant \frac{1}{2} \varepsilon
$$

for all $k$. By passing to a further subsequence we may assume that $\lim _{k \rightarrow \infty} t_{k}=$ $t \in[0, T]$ and $\lim _{k \rightarrow \infty} x_{k}=x \in K$ exist.

Let $\pi_{k}$ denote the equipartition of $\left[0, t_{k}\right]$ into $k$ subintervals of equal length, and note that $\lim _{k \rightarrow \infty} \operatorname{mesh}\left(\pi_{k}\right)=0$. Applying Theorem 3.4 to the sequences $\left(t_{k}\right),\left(x_{k}\right)$ and the partitions $\left(\pi_{k}\right)$, and recalling that $\{\mathscr{P}(t)\}_{t \geqslant 0}$ is $\tau_{c}$-continuous, we see that

$$
\lim _{k \rightarrow \infty} \mathscr{P}\left(t_{k}\right) f\left(x_{k}\right)=\mathscr{P}(t) f(x)=\lim _{k \rightarrow \infty}\left[\mathscr{T}\left(\frac{t_{k}}{n_{k}}\right) \circ \mathscr{S}\left(\frac{t_{k}}{n_{k}}\right)\right]^{n_{k}} f\left(x_{k}\right) .
$$

This contradicts (3.11).

\section{The CaSe When $\{S(t)\}_{t \geqslant 0}$ Restricts to a $C_{0}$-Semigroup on $H_{Q}$}

In this section we will show that condition (3.7) holds whenever the RKHS $H_{Q}$ associated with $Q$ is $\{S(t)\}_{t \geqslant 0}$-invariant and $\{S(t)\}_{t \geqslant 0}$ restricts to a $C_{0}$-semigroup on $H_{Q}$.

Let us fix $t \geqslant 0$ and recall that $Q_{t} \in \mathscr{L}\left(E^{*}, E\right)$ is the positive symmetric operator defined by

$$
Q_{t} x^{*}:=\int_{0}^{t} S(s) Q S^{*}(s) x^{*} d s, \quad x^{*} \in E^{*} .
$$

The RKHS associated with $Q_{t}$ will be denoted by $H_{t}$ and the inclusion operator of $H_{t} \hookrightarrow E$ by $i_{t}$. It is well-known that

$$
H_{t}=\left\{\int_{0}^{t} S(s) i_{Q} f(s) d s: f \in L^{2}\left((0, t) ; H_{Q}\right)\right\}
$$

and that

$$
\|h\|_{H_{t}}=\inf \left\{\|f\|_{L^{2}\left((0, t) ; H_{Q}\right)}: h=\int_{0}^{t} S(s) i_{Q} f(s) d s\right\} .
$$

For Hilbert spaces $E$ this is shown in [7, Appendix B]; the proof carries over to the Banach space case without difficulty. 
Given a partition $P=\left\{t_{0}, \ldots, t_{N}\right\}$ of the interval $[0, t]$, we define the positive symmetric operator $Q_{t}^{P} \in \mathscr{L}\left(E^{*}, E\right)$ as before by

$$
Q_{t}^{P} x^{*}:=\sum_{j=1}^{N}\left(t_{j}-t_{j-1}\right) S\left(t_{j}\right) Q S^{*}\left(t_{j}\right) x^{*}, \quad x^{*} \in E^{*} .
$$

Let $H_{t}^{P}$ denote the associated RKHS with inclusion mapping $i_{t}^{P}: H_{t}^{P} \hookrightarrow E$. Define

$$
\mathscr{H}_{t}^{P}:=\left\{\sum_{j=1}^{N} \int_{t_{j-1}}^{t_{j}} S\left(t_{j}\right) i_{Q} f(s) d s: f \in L^{2}\left((0, t) ; H_{Q}\right)\right\} .
$$

Endowed with the norm

$$
\|h\|_{\mathscr{H}_{t}^{P}}:=\inf \left\{\|f\|_{L^{2}\left((0, t) ; H_{Q}\right)}: h=\sum_{j=1}^{N} \int_{t_{j-1}}^{t_{j}} S\left(t_{j}\right) i_{Q} f(s) d s\right\},
$$

it is easy to see that $\mathscr{H}_{t}^{P}$ is a separable real Hilbert space.

Lemma 4.1. For all $x^{*} \in E^{*}$ we have $Q_{t}^{P} x^{*} \in \mathscr{H}_{t}^{P}$ and

$$
\left\|Q_{t}^{P} x^{*}\right\|_{\mathscr{H}_{t}^{P}} \leqslant\left\|Q_{t}^{P} x^{*}\right\|_{H_{t}^{P}} .
$$

Proof. Fix an arbitrary $x^{*} \in E^{*}$ and define $f_{x^{*}} \in L^{2}\left((0, t) ; H_{Q}\right)$ by

$$
f_{x^{*}}(s):=i_{Q}^{*} S^{*}\left(t_{j}\right) x^{*}, \quad s \in\left(t_{j-1}, t_{j}\right), j=1, \ldots, N .
$$

Then, using that $i_{Q} \circ i_{Q}^{*}=Q$, we have

$$
\sum_{j=1}^{N} \int_{t_{j-1}}^{t_{j}} S\left(t_{j}\right) i_{Q} f_{x^{*}}(s) d s=\sum_{j=1}^{N}\left(t_{j}-t_{j-1}\right) S\left(t_{j}\right) Q^{*} S^{*}\left(t_{j}\right) x^{*}=Q_{t}^{P} x^{*} .
$$

This shows that $Q_{t}^{P} x^{*} \in \mathscr{H}_{t}^{P}$. Furthermore,

$$
\begin{aligned}
& \left\|Q_{t}^{P} x^{*}\right\|_{H_{t}^{P}}^{2}=\left\langle Q_{t}^{P} x^{*}, x^{*}\right\rangle \\
& =\sum_{j=1}^{N}\left(t_{j}-t_{j-1}\right)\left\langle Q S^{*}\left(t_{j}\right) x^{*}, S^{*}\left(t_{j}\right) x^{*}\right\rangle=\sum_{j=1}^{N}\left(t_{j}-t_{j-1}\right)\left\|i_{Q}^{*} S^{*}\left(t_{j}\right) x^{*}\right\|_{H_{Q}}^{2} .
\end{aligned}
$$

Hence,

$$
\left\|Q_{t}^{P} x^{*}\right\|_{\mathscr{H}_{t}^{P}}^{2} \leqslant\left\|f_{x^{*}}\right\|_{L^{2}\left((0, t) ; H_{Q}\right)}^{2}=\sum_{j=1}^{N}\left(t_{j}-t_{j-1}\right)\left\|i_{Q}^{*} S^{*}\left(t_{j}\right) x^{*}\right\|_{H_{Q}}^{2}=\left\|Q_{t}^{P} x^{*}\right\|_{H_{t}^{P}}^{2}
$$

As a consequence, we see that the identity mapping $Q_{t}^{P} x^{*} \mapsto Q_{t}^{P} x^{*}$ extends uniquely to a linear contraction mapping $I_{t}^{P}: H_{t}^{P} \rightarrow \mathscr{H}_{t}^{P}$. We will see below that $I_{t}^{P}$ is injective.

Lemma 4.2. Suppose that $\{S(t)\}_{t \geqslant 0}$ restricts to a $C_{0}-\operatorname{semigroup}\left\{S_{Q}(t)\right\}_{t \geqslant 0}$ on $H_{Q}$. Then $\mathscr{H}_{t}^{P} \subseteq H_{t}$ as subsets of $E$, and for all $h \in \mathscr{H}_{t}{ }^{P}$ we have

$$
\|h\|_{H_{t}} \leqslant\left(\sup _{s \in[0, t]}\left\|S_{Q}(s)\right\|\right)\|h\|_{\mathscr{H}_{t}^{P} .}
$$


Proof. Let $h \in \mathscr{H}_{t}^{P}$ be arbitrary and fixed, and choose $f \in L^{2}\left((0, t) ; H_{Q}\right)$ such that $h=\sum_{j=1}^{N} \int_{t_{j-1}}^{t_{j}} S\left(t_{j}\right) i_{Q} f(s) d s$. Define $g \in L^{2}\left((0, t) ; H_{Q}\right)$ by

$$
g(s):=S_{Q}\left(t_{j}-s\right) f(s), \quad s \in\left(t_{j-1}, t_{j}\right), j=1, \ldots, N .
$$

Noting that $S\left(t_{j}\right) \circ i_{Q}=S(s) \circ S\left(t_{j}-s\right) \circ i_{Q}=S(s) \circ i_{Q} \circ S_{Q}\left(t_{j}-s\right)$ we have

$$
h=\sum_{j=1}^{N} \int_{t_{j-1}}^{t_{j}} S\left(t_{j}\right) i_{Q} f(s) d s=\sum_{j=1}^{N} \int_{t_{j-1}}^{t_{j}} S(s) i_{Q} g(s) d s=\int_{0}^{t} S(s) i_{Q} g(s) d s \in H_{t} .
$$

This proves the inclusion $\mathscr{H}_{t}^{P} \subseteq H_{t}$. Moreover,

$$
\|h\|_{H_{t}} \leqslant\|g\|_{L^{2}\left((0, t) ; H_{Q}\right)} \leqslant\left(\sup _{s \in[0, t]}\left\|S_{Q}(s)\right\|\right)\|f\|_{L^{2}\left((0, t) ; H_{Q}\right)} .
$$

Taking the infimum over all function $f$ representing $h$ we obtain (4.1).

Putting things together we obtain the following commutative diagram:

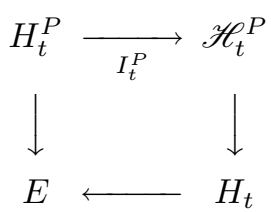

In this diagram, the lower three arrows denote inclusion mappings. Since they are injective, it follows that also $I_{t}^{P}$ is injective and we obtain a (contractive) inclusion mapping $I_{t}^{P}: H_{t}^{P} \hookrightarrow \mathscr{H}_{t}^{P}$. Composing this mapping with the inclusion $\mathscr{H}_{t}^{P} \hookrightarrow H_{t}$ we obtain an inclusion mapping $J_{t}^{P}: H_{t}^{P} \hookrightarrow H_{t}$, which by Lemma 4.2 has norm

$$
\left\|J_{t}^{P}\right\| \leqslant \sup _{s \in[0, t]}\left\|S_{Q}(s)\right\| .
$$

Theorem 4.3. Suppose $\{S(t)\}_{t \geqslant 0}$ restricts to a $C_{0}$-semigroup on $H_{Q}$. Let $\left(t_{n}\right)$ be a sequence of strictly positive real numbers with $\lim _{n \rightarrow \infty} t_{n}=t$. For each $n$ let $P_{n}$ be a partition of $\left[0, t_{n}\right]$, and assume that $\lim _{n \rightarrow \infty} \operatorname{mesh}\left(P_{n}\right)=0$. Then,

$$
\lim _{n \rightarrow \infty} \mu_{t_{n}}^{P_{n}}=\mu_{t} \quad \text { weakly. }
$$

Proof. Choose $T \geqslant 0$ so large that $0 \leqslant t_{n} \leqslant T$ for all $n$. Combination of Lemma $2.4,(3.1)$, and (4.2), shows that for all $n$ we have

$$
\left\langle Q_{t_{n}}^{P_{n}} x^{*}, x^{*}\right\rangle \leqslant\left(\sup _{s \in\left[0, t_{n}\right]}\left\|S_{Q}(s)\right\|\right)^{2}\left\langle Q_{t_{n}} x^{*}, x^{*}\right\rangle \leqslant\left(\sup _{s \in[0, T]}\left\|S_{Q}(s)\right\|\right)^{2}\left\langle Q_{T} x^{*}, x^{*}\right\rangle .
$$

Hence, by Proposition 2.1, the sequence $\left(\mu_{t_{n}}^{P_{n}}\right)$ is tight.

By Lemma 3.3 we have

$$
\lim _{n \rightarrow \infty}\left\langle Q_{t_{n}}^{P_{n}} x^{*}, y^{*}\right\rangle=\left\langle Q_{t} x^{*}, y^{*}\right\rangle
$$

so from (2.2) we conclude that $\mu_{t}$ is the only possible weak limit point of the tight sequence $\left(\mu_{t_{n}}^{P_{n}}\right)$. A standard argument now gives (4.3).

Upon combining this result with Theorem 3.6, we obtain: 
Theorem 4.4. If $\{S(t)\}_{t \geqslant 0}$ restricts to a $C_{0}$-semigroup on $H_{Q}$, then for all $f \in$ $C_{b}(E)$ and all $t \geqslant 0$ and $x \in E$ we have

$$
\mathscr{P}(t) f(x)=\lim _{n \rightarrow \infty}\left[\mathscr{T}\left(\frac{t}{n}\right) \circ \mathscr{S}\left(\frac{t}{n}\right)\right]^{n} f(x),
$$

the convergence being uniform on finite time intervals $[0, T]$ and compact subsets $K \subseteq E$.

\section{The Case When $E$ is a Hilbert space}

In this section we will show that condition (3.7) always holds if $E$ is a separable real Hilbert space.

In the following lemma, $E$ is still allowed to be a separable real Banach space. Recall the standing assumption that $\nu$ is a centred Gaussian measure on $E$ with covariance operator $Q$. For $t \geqslant 0$ let $\rho_{t}$ denote the image measure of $\nu$ under the operator $S(t)$; this is a centred Gaussian measure on $E$ with covariance operator $S(t) Q S^{*}(t)$.

Lemma 5.1. The function $t \mapsto \int_{E}\|x\|^{2} d \rho_{t}(x)$ is continuous on $[0, \infty)$.

Proof. We start by showing that for all $t \geqslant 0$, the family $\left\{\rho_{s}: s \in[0, t]\right\}$ is tight. Fix $\varepsilon>0$ and choose a compact subset $K$ of $E$ with $\nu(K) \geqslant 1-\varepsilon$. Define $L=\{S(s) x: s \in[0, t], x \in K\}$. Being the image of the compact set $[0, t] \times K$ under the continuous mapping $(s, x) \mapsto S(s) x, L$ is compact. For all $s \in[0, t]$ we now have

$$
\rho_{s}(L) \geqslant \rho_{s}(S(s) K)=\nu\{y \in E: S(s) y \in S(s) K\} \geqslant \nu(K) \geqslant 1-\varepsilon .
$$

This proves the asserted tightness.

Fix a nonnegative convergent sequence $\left(t_{n}\right)$ with limit $t$. Consider an arbitrary subsequence $\left(t_{n_{k}}\right)$. The lemma will be proved if we find a further subsequence with the property that

$$
\lim _{j \rightarrow \infty} \int_{E}\|x\|^{2} d \rho_{t_{n_{k_{j}}}}(x)=\int_{E}\|x\|^{2} d \rho_{t}(x) .
$$

By the above, the sequence $\left(\rho_{t_{n_{k}}}\right)$ is tight. Consequently, there is a subsequence $\left(\rho_{t_{n_{k_{j}}}}\right)$ converging weakly to some probability measure $\tilde{\rho}_{t}$. Since the weak limit of a sequence of centred Gaussian measures is a centred Gaussian measure and since

$$
\lim _{j \rightarrow \infty}\left\langle S\left(t_{n_{k_{j}}}\right) Q S^{*}\left(t_{n_{k_{j}}}\right) x^{*}, y^{*}\right\rangle=\left\langle S(t) Q S^{*}(t) x^{*}, y^{*}\right\rangle
$$

for all $x^{*}, y^{*} \in E^{*}$, it follows that $\tilde{\rho}_{t}=\rho_{t}$. Hence, (5.1) follows from Proposition 2.3 , part (i).

Suppose now that $E$ is a separable real Hilbert space. Then, we may identify $Q$ with a positive selfadjoint operator on $E$. Since, by assumption, $Q$ is a Gaussian covariance, $Q$ may be identified with a trace class operator on $E$ and by (2.5), Lemma 5.1 may be reformulated as saying that the function $t \mapsto \operatorname{tr} S(t) Q S^{*}(t)$ is continuous on $[0, \infty)$. Only this fact will be needed below, and it is worthwile to point out that this can be proved more directly as follows. Let $\left(e_{j}\right)$ be an 
orthonormal basis of $E$ and suppose that $t_{n} \rightarrow t$ in $[0, \infty)$. Then,

$$
\begin{aligned}
\lim _{n \rightarrow \infty} \operatorname{tr} S\left(t_{n}\right) Q S^{*}\left(t_{n}\right)=\lim _{n \rightarrow \infty} \sum_{j}\left\|Q^{\frac{1}{2}} S^{*}\left(t_{n}\right) e_{j}\right\|^{2} \\
=\lim _{n \rightarrow \infty} \sum_{j} \sum_{k}\left[Q^{\frac{1}{2}} S^{*}\left(t_{n}\right) e_{j}, e_{k}\right]_{E}^{2}=\lim _{n \rightarrow \infty} \sum_{k}\left\|S\left(t_{n}\right) Q^{\frac{1}{2}} e_{k}\right\|^{2} \\
=\sum_{k}\left\|S(t) Q^{\frac{1}{2}} e_{k}\right\|^{2}=\sum_{k} \sum_{j}\left[S(t) Q^{\frac{1}{2}} e_{k}, e_{j}\right]_{E}^{2} \\
=\sum_{j}\left\|Q^{\frac{1}{2}} S^{*}(t) e_{j}\right\|^{2}=\operatorname{tr} S(t) Q S^{*}(t)
\end{aligned}
$$

the convergence of the series being justified by dominated convergence, since we have, for some constant $C \geqslant 0,\left\|S\left(t_{n}\right) Q^{\frac{1}{2}} e_{j}\right\|^{2} \leqslant C\left\|Q^{\frac{1}{2}} e_{j}\right\|^{2}$, and the latter is a summable sequence:

$$
\sum_{j}\left\|Q^{\frac{1}{2}} e_{j}\right\|^{2}=\operatorname{tr} Q
$$

Theorem 5.2. Let $E$ be a separable real Hilbert space. Let $\left(t_{n}\right)$ be a sequence of strictly positive real numbers with $\lim _{n \rightarrow \infty} t_{n}=t$. For each $n$ let $P_{n}$ be a partition of $\left[0, t_{n}\right]$, and assume that $\lim _{n \rightarrow \infty} \operatorname{mesh}\left(P_{n}\right)=0$. Then,

$$
\lim _{n \rightarrow \infty} \mu_{t_{n}}^{P_{n}}=\mu_{t} \text { weakly. }
$$

Proof. By Lemma 3.3, for all $x, y \in E$ we have

$$
\lim _{n \rightarrow \infty}\left[Q_{t_{n}}^{P_{n}} x, y\right]_{E}=\left[Q_{t} x, y\right]_{E} .
$$

Hence, by part (ii) of Proposition 2.3, it remains to check that

$$
\lim _{n \rightarrow \infty} \int_{E}\|x\|^{2} d \mu_{t_{n}}^{P_{n}}(x)=\int_{E}\|x\|^{2} d \mu_{t}(x) .
$$

This is equivalent to the condition

$$
\lim _{n \rightarrow \infty} \operatorname{tr} Q_{t_{n}}^{P_{n}}=\operatorname{tr} Q_{t} .
$$

Choose an orthonormal basis $\left\{e_{k}\right\}_{k=1}^{\infty}$ for $E$. Then,

$$
\begin{aligned}
\operatorname{tr} Q_{t_{n}}^{P_{n}} & =\sum_{k=1}^{\infty} \sum_{j=1}^{N_{n}}\left(t_{j, n}-t_{j-1, n}\right)\left[S\left(t_{j, n}\right) Q S^{*}\left(t_{j, n}\right) e_{k}, e_{k}\right]_{E} \\
& =\sum_{j=1}^{N_{n}}\left(t_{j, n}-t_{j-1, n}\right) \sum_{k=1}^{\infty}\left[S\left(t_{j, n}\right) Q S^{*}\left(t_{j, n}\right) e_{k}, e_{k}\right]_{E} \\
& =\sum_{j=1}^{N_{n}}\left(t_{j, n}-t_{j-1, n}\right) \operatorname{tr} S\left(t_{j, n}\right) Q S^{*}\left(t_{j, n}\right),
\end{aligned}
$$

where the change in the order of summation is justified by the fact that each term $\left[S\left(t_{j, n}\right) Q S^{*}\left(t_{j, n}\right) e_{k}, e_{k}\right]_{E}=\left[Q S^{*}\left(t_{j, n}\right) e_{k}, S^{*}\left(t_{j, n}\right) e_{k}\right]_{E}$ is nonnegative.

The right hand side of (5.2) is a Riemann sum of the integral

$$
\int_{0}^{t_{n}} \operatorname{tr} S(s) Q S(s) d s
$$


As we noted, by Lemma 5.1 the function $s \mapsto \operatorname{tr} S(s) Q S(s)$ is continuous on $[0, t]$. Arguing as in the proof of Lemma 3.3, this implies

$$
\limsup _{n \rightarrow \infty}\left|\operatorname{tr} Q_{t_{n}}^{P_{n}}-\int_{0}^{t} \operatorname{tr} S(s) Q S(s) d s\right|<\varepsilon
$$

for all $\varepsilon>0$. Hence,

$$
\lim _{n \rightarrow \infty} \operatorname{tr} Q_{t_{n}}^{P_{n}}=\int_{0}^{t} \operatorname{tr} S(s) Q S(s) d s=\operatorname{tr} Q_{t} .
$$

Upon combining this result with Theorem 3.6, we obtain:

Corollary 5.3. If $E$ is a separable real Hilbert space, then for all $f \in C_{b}(E)$ and all $t \geqslant 0$ and $x \in E$ we have

$$
\mathscr{P}(t) f(x)=\lim _{n \rightarrow \infty}\left[\mathscr{T}\left(\frac{t}{n}\right) \circ \mathscr{S}\left(\frac{t}{n}\right)\right]^{n} f(x),
$$

the convergence being uniform on finite time intervals $[0, T]$ and compact subsets $K \subseteq E$.

For separable real Hilbert spaces $E$, a Lie-Trotter product formula for a class of transition semigroups on $C_{b}(E)$ associated with nonlinear stochastic equations of the form

$$
\begin{aligned}
d X(t) & =F(X(t)) d t+B(X(t)) d W(t), \quad t \geqslant 0, \\
X(0) & =x_{0},
\end{aligned}
$$

has been obtained recently by G. Tessitore and J. Zabczyk. Here, $\{W(t)\}_{t \geqslant 0}$ is a Brownian motion with values in $E$, and $F: E \rightarrow E$ and $B: E \rightarrow \mathscr{L}(E)$ are Lipschitz functions. In the linear case there is a small overlap with our Corollary 5.3. To make this explicit we make two special choices of $F$ and $B$ in (5.3). First, we let $\mathscr{T}$ be the transition semigroup on $C_{b}(E)$ obtained by taking $F \equiv A$, with $A$ a given bounded operator on $E$, and $B \equiv 0$ in (5.3); thus,

$$
\mathscr{T}(t) f(x)=f\left(e^{t A} x\right), \quad t \geqslant 0, x \in E .
$$

Second, we let $\mathscr{S}$ be the transition semigroup on $C_{b}(E)$ obtained by taking $F \equiv$ $0 \in \mathscr{L}(E)$ and $B \equiv I$ in (5.3); thus,

$$
\mathscr{S}(t) f(x)=\int_{E} f(x+y) d \mu_{t}(y), \quad t \geqslant 0, x \in E,
$$

where $\mu_{t}$ is the distribution of $W(t)$. This puts us into the setting considered in Corollary 5.3. From [17, Proposition 3.5] (the special case for uniformly bounded $F$ and $B$ of the main result, [17, Theorem 3.4]) we now see the following. Let $Y$ denote the closure with respect to the supremum norm in $C_{b}(E)$ of the space of all functions which are bounded and uniformly continuous along with their first and second Fréchet derivatives. Then for all $f \in Y, t \geqslant 0$, and $x \in E$,

$$
\mathscr{P}(t) f(x)=\lim _{n \rightarrow \infty}\left[\mathscr{T}\left(\frac{t}{n}\right) \circ \mathscr{S}\left(\frac{t}{n}\right)\right]^{n} f(x),
$$

the convergence being uniform on finite time intervals and bounded subsets $B \subseteq E$. 


\section{Appendix: Proof of Proposition 2.3, part (ii)}

Although part (ii) of Proposition 2.3 may be well-known to specialists, we could not find an explicit reference for it, and for the convenience of the reader we include a proof here.

Let $E$ be a separable real Hilbert space and let $\left(\mu_{n}\right)_{n=1}^{\infty}$ and $\mu$ satisfy the conditions (1) and (2) in Proposition 2.3. We choose an orthonormal basis $\left\{e_{j}\right\}_{j=1}^{\infty}$ of $E$ and denote by $P_{j}$ the orthogonal projection onto the linear span of $\left\{e_{1}, \ldots, e_{j}\right\}$.

Lemma 6.1. Let $\varepsilon>0$ be arbitrary and fixed. For all $k \geqslant 1$ there exists an index $J_{k}$ with the following property: for all $j \geqslant J_{k}$ and all $n \geqslant 1$ we have

$$
\mu_{n}\left\{x \in E:\left\|x-P_{j} x\right\|^{2}>\frac{1}{k}\right\} \leqslant \frac{\varepsilon}{2^{k+1}} .
$$

Proof. The proof is inspired by an argument in [1].

Denote $\mu_{n, j}:=P_{j} \mu_{n}$ and $\mu_{j}:=P_{j} \mu$, and let $Q_{n, j}$ and $Q_{j}$ denote their covariance operators. By condition (1), for all $x, y \in E$ we have

$$
\begin{aligned}
\lim _{n \rightarrow \infty}\left[Q_{n, j} x, y\right]_{E}=\lim _{n \rightarrow \infty}[ & \left.P_{j} Q_{n} P_{j} x, y\right]_{E}=\lim _{n \rightarrow \infty}\left[Q_{n} P_{j} x, P_{j} y\right]_{E} \\
=\left[Q P_{j} x, P_{j} y\right]_{E} & =\left[P_{j} Q_{j} P_{j} x, y\right]=\left[Q_{j} x, y\right]_{E} .
\end{aligned}
$$

Hence by (2.1),

$$
\begin{aligned}
\lim _{n \rightarrow \infty} \int_{E} & \left\|P_{j} x\right\|^{2} d \mu_{n}(x)=\lim _{n \rightarrow \infty} \int_{P_{j} E}\|y\|^{2} d \mu_{n, j}(y) \\
& =\lim _{n \rightarrow \infty} \sum_{k=1}^{j} \int_{P_{j} E}\left[y, e_{k}\right]_{E}^{2} d \mu_{n, j}(y)=\lim _{n \rightarrow \infty} \sum_{k=1}^{j}\left[Q_{n, j} e_{k}, e_{k}\right]_{E} \\
& =\sum_{k=1}^{j}\left[Q_{j} e_{k}, e_{k}\right]_{E}=\sum_{k=1}^{j} \int_{P_{j} E}\left[y, e_{k}\right]_{E}^{2} d \mu_{j}(y) \\
& =\int_{P_{j} E}\|y\|^{2} d \mu_{j}(y)=\int_{E}\left\|P_{j} x\right\|^{2} d \mu(x) .
\end{aligned}
$$

By the absolute continuity of the measure $\|x\|^{2} d \mu(x)$ with respect to $d \mu(x)$, for every integer $k \geqslant 1$ we can pick $\delta_{k}>0$ such that

$$
\int_{A}\|x\|^{2} d \mu(x) \leqslant \frac{\varepsilon}{k 2^{k+3}}
$$

for all Borel sets $A \subseteq E$ with $\mu(A) \leqslant \delta_{k}$. Define

$$
A_{j, k}:=\left\{x \in E:\left\|x-P_{j} x\right\|^{2}>\frac{\varepsilon}{k 2^{k+3}}\right\} .
$$

By dominated convergence we have $\lim _{j \rightarrow \infty} \mu\left(A_{j, k}\right)=0$ for all $k \geqslant 1$. It follows that there exists an index $J(k)$ such that $\mu\left(A_{j, k}\right) \leqslant \delta_{k}$ for all $j \geqslant J(k)$. Then, for all $j \geqslant J(k)$ we have

$$
\begin{gathered}
\int_{E}\|x\|^{2}-\left\|P_{j} x\right\|^{2} d \mu(x)=\int_{E}\left\|x-P_{j} x\right\|^{2} d \mu(x) \\
\leqslant \int_{A_{j, k}}\|x\|^{2} d \mu(x)+\int_{E \backslash A_{j, k}}\left\|x-P_{j} x\right\|^{2} d \mu(x) \\
\leqslant \frac{\varepsilon}{k 2^{k+3}}+\frac{\varepsilon}{k 2^{k+3}}=\frac{\varepsilon}{k 2^{k+2}} .
\end{gathered}
$$


Next, choose an index $N_{k}$ so large that for all $n \geqslant N_{k}$ we have

$$
\left|\int_{E}\|x\|^{2} d \mu_{n}(x)-\int_{E}\|x\|^{2} d \mu(x)\right| \leqslant \frac{\varepsilon}{k 2^{k+3}}
$$

and

$$
\left|\int_{E}\left\|P_{J(k)} x\right\|^{2} d \mu(x)-\int_{E}\left\|P_{J(k)} x\right\|^{2} d \mu_{n}(x)\right| \leqslant \frac{\varepsilon}{k 2^{k+3}} .
$$

The second condition can be met in view of (6.1). Then, for all $n \geqslant N_{k}$ and $j \geqslant J(k)$,

$$
\begin{gathered}
\int_{E}\left\|x-P_{j} x\right\|^{2} d \mu_{n}(x) \leqslant \int_{E}\left\|x-P_{J(k)} x\right\|^{2} d \mu_{n}(x)=\int_{E}\|x\|^{2}-\left\|P_{J(k)} x\right\|^{2} d \mu_{n}(x) \\
\leqslant\left|\int_{E}\|x\|^{2} d \mu_{n}(x)-\int_{E}\|x\|^{2} d \mu(x)\right|+\int_{E}\|x\|^{2}-\left\|P_{J(k)} x\right\|^{2} d \mu(x) \\
\quad+\left|\int_{E}\left\|P_{J(k)} x\right\|^{2} d \mu_{n}(x)-\int_{E}\left\|P_{J(k)} x\right\|^{2} d \mu(x)\right| \\
\leqslant \frac{\varepsilon}{k 2^{k+3}}+\frac{\varepsilon}{k 2^{k+2}}+\frac{\varepsilon}{k 2^{k+3}}=\frac{\varepsilon}{k 2^{k+1}} .
\end{gathered}
$$

It follows that for all $n \geqslant N_{k}$ and all $j \geqslant J(k)$ we have

$$
\mu_{n}\left\{x \in E:\left\|x-P_{j} x\right\|^{2}>\frac{1}{k}\right\} \leqslant k \int_{E}\left\|x-P_{j} x\right\|^{2} d \mu_{n}(x) \leqslant \frac{\varepsilon}{2^{k+1}} .
$$

By dominated convergence, for every $k \geqslant 1$ we can find an index $J_{k} \geqslant J(k)$ such that for all $n=1, \ldots, N_{k}-1$ and all $j \geqslant J_{k}$ we have

$$
\mu_{n}\left\{x \in E:\left\|x-P_{j} x\right\|^{2}>\frac{1}{k}\right\} \leqslant \frac{\varepsilon}{2^{k+1}} .
$$

This $J_{k}$ has the desired properties.

Proof of Proposition 2.3, part (ii). We follow the argument of [18, Theorem I.3.7]. Define

$$
V_{j, k}:=\left\{x \in E:\left\|x-P_{j} x\right\|^{2}>\frac{1}{k}\right\} .
$$

Fix $\varepsilon>0$ arbitrary. By Lemma 6.1 , for every $k \geqslant 1$ we can find an index $j_{k}$ such that for all $n \geqslant 1$ we have

$$
\mu_{n}\left(V_{j_{k}, k}\right) \leqslant \frac{\varepsilon}{2^{k+1}} .
$$

For all $n \geqslant 1$ and $r>0$ we have

$$
\mu_{n}\{x \in E:\|x\|>r\} \leqslant \frac{1}{r^{2}} \int_{E}\|x\|^{2} d \mu_{n}(x) \leqslant \frac{1}{r^{2}} \cdot \sup _{m \geqslant 1} \int_{E}\|x\|^{2} d \mu_{m}(x) .
$$

Hence, we may choose $r_{0}$ so large that for all $n \geqslant 1$ we have

Set

$$
\mu_{n}\left\{x \in E:\|x\|>r_{0}\right\} \leqslant \frac{\varepsilon}{2} .
$$

$$
F:=\left(\bigcap_{k \geqslant 1} E \backslash V_{j_{k}, k}\right) \bigcap\left\{x \in E:\|x\| \leqslant r_{0}\right\} .
$$

Then, $F$ is bounded and closed, and for all $k \geqslant 1$ we have

$$
F \subseteq\left\{x \in E:\left\|x-P_{j_{k}} x\right\|^{2} \leqslant \frac{1}{k}\right\} .
$$


Since every $P_{j_{k}}$ has finite-dimensional range, an elementary argument implies that $F$ is compact. Moreover, for all $n \geqslant 1$ we have

$$
\mu_{n}(E \backslash F) \leqslant\left(\sum_{k=1}^{\infty} \frac{\varepsilon}{2^{k+1}}\right)+\frac{\varepsilon}{2} \leqslant \varepsilon
$$

We have shown that for every $\varepsilon>0$ there exists a compact set $F \subseteq E$ with $\mu_{n}(F) \geqslant 1-\varepsilon$ for $n \geqslant 1$. This proves that the sequence $\left(\mu_{n}\right)$ is tight.

By condition (1) and (2.2), $\mu$ is the only possible weak limit point of $\left(\mu_{n}\right)$. A standard argument now gives the weak convergence $\lim _{n \rightarrow \infty} \mu_{n}=\mu$.

Acknowledgement - We gratefully acknowledge the valuable suggestions of the anonymous referees.

\section{REFERENCES}

[1] A.N. Baushev, On weak convergence of Gaussian measures, Prob. Teor. Veroyatnost. i Primenen. 32 (1987), 734-742. English translation in: Theory Probab. Appl. 32 (1987), 670-677.

[2] V.I. Bogachev, "Gaussian Measures", Math. Surveys and Monographs, Vol. 62, Amer. Math. Soc., Providence, RI, 1998.

[3] Z. BrzeźniaK, On stochastic convolution in Banach spaces and applications, Stoch. Stoch. Reports 61 (1997), 245-295.

[4] Z. Brzeźniak and J.M.A.M. van Neerven, Stochastic convolution in separable Banach spaces and the stochastic linear Cauchy problem, Studia Math. 143 (2000), 43-74.

[5] S. Cerrai, A Hille-Yosida theorem for weakly continuous semigroups, Semigroup Forum 49 (1994), 349-367.

[6] S. Cerrai And F. Gozzi, Strong solutions of Cauchy problems associated to weakly continuous semigroups, Diff. Integral Eq. 8 (1995), 465-486.

[7] G. Da Prato and J. ZabczyK, "Stochastic Equations in Infinite Dimensions", Encyclopedia of Mathematics and its Applications, Cambridge University Press, Cambridge, 1992.

[8] B. Goldys And M. Kocan, Diffusion semigroups in spaces of continuous functions with mixed topology, J. Differential Equations 173 (2001), 17-39.

[9] B. Goldys and J.M.A.M. van Neerven, Transition semigroups of Ornstein-Uhlenbeck processes on Banach spaces, submitted.

[10] F. Kühnemund, A Hille-Yosida theorem for bi-continuous semigroups, Semigroup Forum, to appear.

[11] F. Kühnemund, Approximation of bi-continuous semigroups, submitted.

[12] J.M.A.M. van NeERVEn, Nonsymmetric Ornstein-Uhlenbeck semigroups in Banach spaces, J. Funct. Anal. 155 (1998), 495-535.

[13] J.M.A.M. van NeERven, Continuity and representation of Gaussian Mehler semigroups, Potential Anal. 13 (2000), 199-211.

[14] J.M.A.M. van NeERven and L. Weis, Weak operator limits and integrals of Gaussian covariances in Banach spaces, preprint.

[15] J.M.A.M. VAN NEERVEn AND J. ZABCZYK, Norm discontinuity of Ornstein-Uhlenbeck semigroups, Semigroup Forum 59 (1999), 389-403.

[16] E. Priola, On a class of Markov type semigroups in spaces of uniformly continuous and bounded functions, Studia Math. 136 (1999), 271-295.

[17] G. Tessitore and J. ZabCZyK, Trotter's formula for transition semigroups, Semigroup Forum 63 (2001), 114-126.

[18] N.N. Vakhania, V.I. Tarieladze, and S.A. Chobanyan, "Probability Distributions in Banach Spaces", D. Reidel Publishing Company, Dordrecht-Boston-Lancaster-Tokyo, 1987. 
Mathematisches Institut, Universität Tübingen, Auf der Morgenstelle 10, D-72076 GERMANY

E-mail address: frku@giotto.mathematik.uni-tuebingen.de

Department of Applied Mathematical Analysis, Technical University of Delft, P.O. Box 5031, 2600 GA Delft, The Netherlands

E-mail address: J.vanNeerven@its.tudelft.nl 